\title{
Restoration of HPA axis is rapid in subclinical Cushing's syndrome after adrenalectomy
}

Hee Kyung Kim, Yunah Jeong, Jee Hee Yoon, Ho-Cheol Kang

Department of Internal Medicine, Chonnam National University Medical School, Gwangju, South Korea

\section{INTRODUCTION}

- Subclinical Cushing's syndrome (SC) is a status of cortisol excess defined by dynamic hormonal tests in patients with adrenal incidentaloma who have no overt syndrome of Cushing's syndrome.

- SC is assumed that cortisol production is insufficient to cause a clinically recognizable syndrome and to suppress the release of $\mathrm{CRH}$ and ACTH.

- Differences in hormonal levels or recovery time of the hypothalamicpituitary-adrenocortical (HPA) axis after adrenalectomy between patients with overt Cushing's syndrome $(\mathrm{OC})$ and SC remain unknown.

\section{METHOD}

- Thirty-six patients (10 with OC and 26 with SC) with adrenal Cushing's syndrome who underwent adrenalectomy from 2004 to 2014 were reviewed retrospectively.

- Patients were treated with glucocorticoid after adrenalectomy and were reevaluated at discharge and every 1 months thereafter using a morning cortisol or rapid ACTH stimulation test.

\section{RESULTS}

Adrenal incidentaloma $(n=674)$

Overnight dexamethasone suppression test(DST): cortisol $<1.8 \mu \mathrm{g} / \mathrm{dL}$ $24 \mathrm{hr}$ urine free cortisol $<50 \mu \mathrm{g} /$ day Low/high DST: cortisol $<1.8 \mu \mathrm{g} / \mathrm{dL}$

\section{NO}

Subclinical Cushing's syndrome $(n=38)$

Adrenalectomy

\section{YES}

Enrolled subclinical Cushing's syndrome $(n=26)$

Overt Cushing's syndrome $(n=10)$

Figure 1. Study design.

Table 2. Relationship between recovery time and preoperative cortisol levels

\begin{tabular}{|c|c|c|c|c|c|}
\hline & $\begin{array}{l}\text { Recovery time } \\
\text { (months) }\end{array}$ & \multicolumn{2}{|c|}{$\begin{array}{c}\text { Basal UFC } \\
\text { ( } \mu \mathrm{g} / \mathrm{day})\end{array}$} & \multicolumn{2}{|c|}{$\begin{array}{c}\text { P-cortisol after ODST } \\
(\mu \mathrm{g} / \mathrm{dL})\end{array}$} \\
\hline Median (range) & $5(0-30)$ & \multicolumn{2}{|c|}{$74.0(26-1450.0)$} & & $.4(2.1-24.1)$ \\
\hline$\rho \dagger$ & & \multicolumn{2}{|c|}{$0.485^{*}$} & & $0.645^{* \star}$ \\
\hline $\mathbf{R}^{2} \ddagger$ & & \multicolumn{2}{|c|}{$0.197^{*}$} & & $0.465^{* *}$ \\
\hline & $\begin{array}{l}\text { UFC after LDST } \\
(\mu \mathrm{g} / \mathrm{day})\end{array}$ & $\begin{array}{l}\text { P-cortisol after } \\
\text { LDST ( } \mu \mathrm{g} / \mathrm{dL} \text { ) }\end{array}$ & \multicolumn{2}{|c|}{$\begin{array}{l}\text { UFC after HDST } \\
\text { ( } \mu \text { g/day) }\end{array}$} & $\begin{array}{l}\text { P-cortisol after } \\
\text { HDST }(\mu \mathrm{g} / \mathrm{dL})\end{array}$ \\
\hline Median (range) & $48.3(5.0-830.0)$ & $11.1(2.7-29.7)$ & \multicolumn{2}{|c|}{$57.0(9.0-963.0)$} & $10.3(2.2-39.1)$ \\
\hline p† & $0.729^{* *}$ & $0.688^{* *}$ & \multicolumn{2}{|c|}{$0.697^{* *}$} & $0.606^{* *}$ \\
\hline $\mathbf{R}^{2} \ddagger$ & $0.598^{* *}$ & $0.472^{* *}$ & \multicolumn{2}{|c|}{$0.495^{\star *}$} & $0.303^{*}$ \\
\hline
\end{tabular}

UFC, 24-h urine free cortisol; P-cortisol, plasma cortisol; ODST, $1 \mathrm{mg}$ overnight dexamethasone suppression test; LDST, $4 \mathrm{mg}$ low-dose dexamethasone suppression test; HDST, $16 \mathrm{mg}$ high-dose dexamethasone suppression tes

${ }^{*} p<0.05$

${ }^{* *} p<0.001$

† $\rho$ (Spearman's rho) represents the correlation coefficient between recovery time and the clinical parameters of cortisol excretion

$\ddagger R^{2}$ was calculated by linear regression analysis
Table 1. Baseline characteristics of patients with subclinical and overt Cushing's syndrome

\begin{tabular}{|c|c|c|c|}
\hline & $\begin{array}{l}\text { Overt } \\
(N=10)\end{array}$ & $\begin{array}{l}\text { Subclinical } \\
\quad(\mathrm{N}=26)\end{array}$ & $p$ value \\
\hline Age (years) & $47.3 \pm 12.3$ & $56.0 \pm 9.3$ & 0.028 \\
\hline Gender (Female, \%) & $9(90.0)$ & $19(73.1)$ & 0.274 \\
\hline BMI, $\mathrm{kg} / \mathrm{m}^{2}$ & $23.7 \pm 3.2$ & $24.6 \pm 3.9$ & 0.391 \\
\hline Hypertension (\%) & $7(70.0)$ & $11(42.3)$ & 0.137 \\
\hline Diabetes mellitus (\%) & $5(50.0)$ & $7(26.9)$ & 0.188 \\
\hline Obesity (\%)* & $6(60.0)$ & $17(65.4)$ & 0.763 \\
\hline Tumor size $(\mathrm{cm})$ & $2.7 \pm 0.7$ & $2.4 \pm 0.9$ & 0.348 \\
\hline Tumor location (Right, \%) & $2(20.0)$ & $7(26.9)$ & 0.667 \\
\hline ACTH, pg/mL & $11.7 \pm 6.0$ & $24.3 \pm 16.1$ & 0.023 \\
\hline Basal UFC, $\mu \mathrm{g} / \mathrm{day}$ & $520.1 \pm 432.0$ & $71.9 \pm 33.1$ & 0.010 \\
\hline Cortisol after ODST, $\mu \mathrm{g} / \mathrm{dL}$ & $18.1 \pm 6.0$ & $7.8 \pm 5.9$ & 0.001 \\
\hline UFC after LDST, $\mu \mathrm{g} / \mathrm{day}$ & $472.8 \pm 208.4$ & $56.8 \pm 77.7$ & $<0.001$ \\
\hline Cortisol after LDST, $\mu \mathrm{g} / \mathrm{dL}$ & $22.2 \pm 6.2$ & $8.7 \pm 6.1$ & $<0.001$ \\
\hline UFC after HDST, $\mu \mathrm{g} /$ day & $487.8 \pm 284.2$ & $74.1 \pm 113.5$ & 0.001 \\
\hline Cortisol after HDST, $\mu \mathrm{g} / \mathrm{dL}$ & $21.1 \pm 5.8$ & $10.2 \pm 9.0$ & 0.001 \\
\hline DHEA-S, $\mu \mathrm{g} / \mathrm{dL}$ & $20.3 \pm 14.1$ & $43.5 \pm 71.7$ & 0.490 \\
\hline $\begin{array}{l}\text { Recovery time (median, range, } \\
\text { months) }\end{array}$ & $17.0(5-30)$ & $4.0(0-14)$ & $<0.001$ \\
\hline
\end{tabular}

BMI, body mass index; UFC, 24-h urine free cortisol; ODST, overnight dexamethasone suppression test: LDST, low-dose dexamethasone suppression test; HDST, high-dose dexamethasone suppression tes

* Description of the criteria for obesity

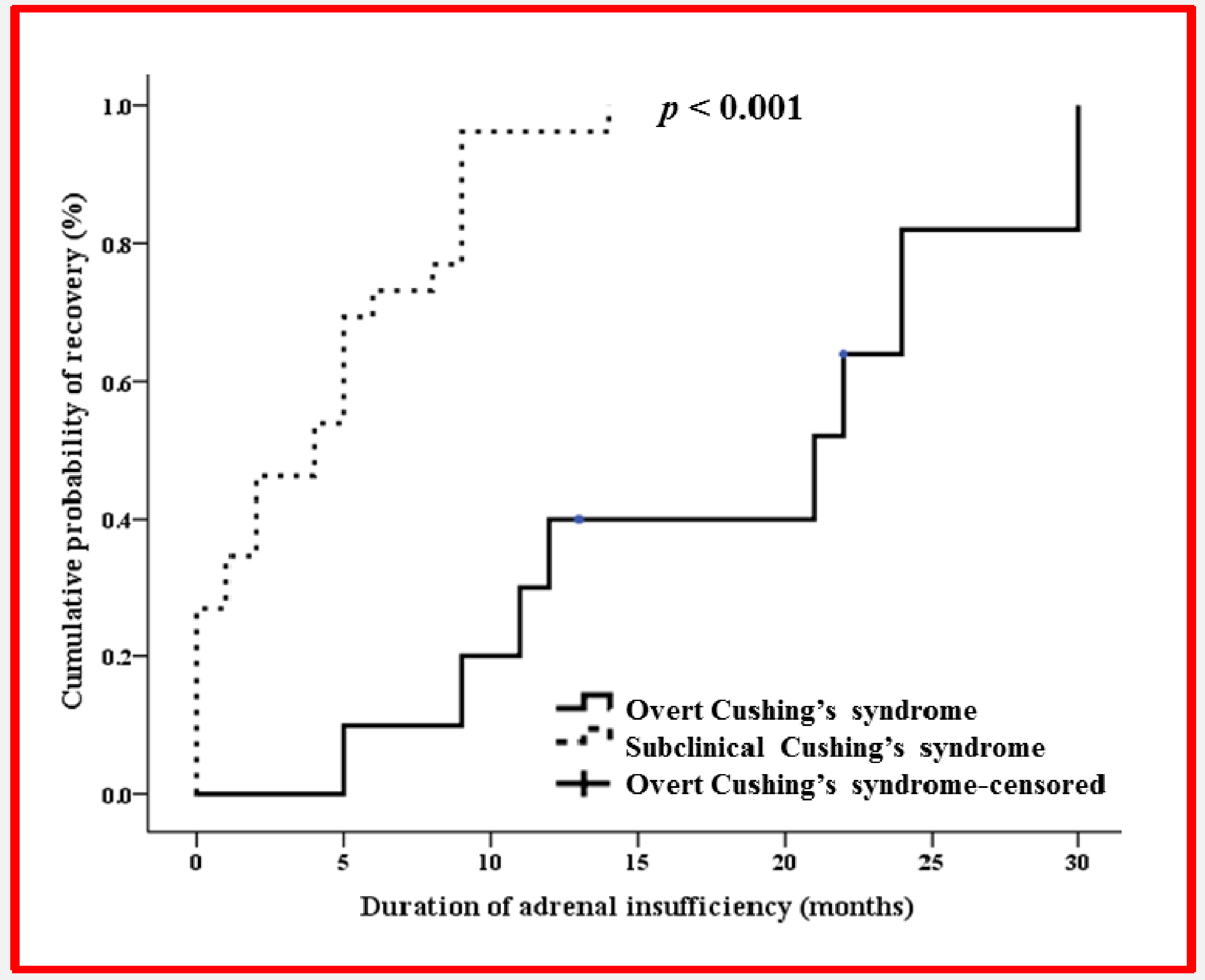

Figure 2. Cumulative probability of adrenal function recovery in patients with overt and subclinical Cushing's syndrome

\section{CONCLUSION}

The degree of cortisol excess differs between the two disease categories, and the time to adrenal function recovery was associated with the degree of cortisol excess.

- The HPA axis recovery time after adrenalectomy in patients with SC is rapid (within several months) and is dependent on the degree of cortisol excess.

Routine postoperative glucocorticoid replacement is not necessary in some patients with SC. 